\title{
PERFORMANCE LIMITATIONS IN CONTROL SYSTEMS WITH SENSOR TIME DELAYS
}

\author{
Daniel E. Davison $^{* 1}$ Robert Tonita * \\ * Department of Electrical and Computer Engineering, \\ University of Waterloo, Canada, N2L 3G1
}

\begin{abstract}
This paper derives performance limitations associated with sensor delays in single-input single-output discrete-time feedback loops. Aspects of performance considered are tracking and sensitivity to plant uncertainty. It is shown that, in both one degreeof-freedom and two degree-of-freedom control configurations, closed-loop sensitivity with respect to plant model uncertainty is fundamentally limited by the presense of the sensor delay. The sensitivity bound provided in this paper can be used to quantify how sensitivity necessarily worsens as the sensor delay increases and as the closed-loop bandwidth increases. Copyright $\left.{ }^{(}\right)_{2005}$ IFAC
\end{abstract}

Keywords: Time delay, delay compensation, performance limits, observers, discrete-time systems.

\section{INTRODUCTION}

Recently there has been an initiative to incorporate feedback control into radiotherapy, i.e., the treatment of cancer by external ionizing radiation (Davison and Hwang, 2003). The radiotherapy research project has motivated several theoretical control problems, including the question of how to accommodate time delays in feedback sensors. In the case of the radiotherapy work, sensor delays arise because of image processing and calculations required to determine tumor position and radiation dose distribution information; sensor delays arise naturally in other applications where, for example, data must be transmitted over huge distances.

To explain the sensor delay compensation problem addressed in this paper, consider the three block diagrams in Figure 1. Figure 1(a) shows the classical one-degree-of-freedom (1-DOF) single-input singleoutput (SISO) unity-feedback control topology. The plant and controller are linear time-invariant (LTI)

\footnotetext{
1 Funded by the Natural Sciences and Engineering Research Council of Canada (NSERC).
}

with discrete-time transfer functions $P[z]$ and $C^{a}[z]$, respectively, where the superscript $a$ is used to connect the controller with the block diagram in Figure 1(a). Partly for simplicity and partly because of technical hurdles, it will be assumed throughout that the plant, $P[z]$, is stable; this is consistent with the motivating radiotherapy example. It is also assumed that the plant $P[z]$ is not identically zero, which is a trivial and uninteresting case. Using standard notation, $r[k]$ denotes the reference signal, $y[k]$ denotes the plant output, and $e[k]:=r[k]-y[k]$ denotes the tracking error. In Figure 1(a) it is assumed that the sensor is perfect, i.e., it has no delay. It is also assumed that $C^{a}[z]$ has been designed to achieve desired closed-loop properties. In addition to stability, the closed-loop properties in question are as follows, where $T_{\alpha \beta}^{a}$ denotes the transfer function from signal $\alpha$ to signal $\beta$ (with the superscript $a$ again used to connect the transfer functions with Figure 1(a)), and where the discrete-time frequency is denoted $\Omega$ :

- Good tracking, as measured by the magnitude of $T_{r e}^{a}\left[e^{j \Omega}\right]=1 /\left(1+P\left[e^{j \Omega}\right] C^{a}\left[e^{j \Omega}\right]\right)$. 
- Good sensitivity of $T_{r y}^{a}$ with respect to perturbations in the plant, $P[z]$, as measured by the magnitude of

$$
S^{a}\left[e^{j \Omega}\right]:=\frac{\partial T_{r y}^{a}\left[e^{j \Omega}\right]}{\partial P\left[e^{j \Omega}\right]} \frac{P\left[e^{j \Omega}\right]}{T_{r y}^{a}\left[e^{j \Omega}\right]}=\frac{1}{1+P\left[e^{j \Omega}\right] C^{a}\left[e^{j \Omega}\right]} .
$$

Typically, it is desired to achieve "good" tracking and "good" sensitivity at low frequencies; at high frequencies, the loop gain must be rolled off due to other concerns, e.g., sensor noise and plant model uncertainty. It is popular to express this type of requirement using weighting functions and $\infty$-norms. For the purposes of this paper, assume there exist stable, biproper (i.e., relative degree zero), low-pass weights $W_{1}[z], W_{2}[z]$ and positive constants $\varepsilon_{1}, \varepsilon_{2}$ such that "good" performance is equivalent to closed-loop stability and

$$
\begin{aligned}
\left\|W_{1} T_{r e}^{a}\right\|_{\infty} & :=\max _{0 \leq \Omega<\pi}\left|W_{1}\left[e^{j \Omega}\right] T_{r e}^{a}\left[e^{j \Omega}\right]\right| \leq \varepsilon_{1}, \\
\left\|W_{2} S^{a}\right\|_{\infty} & :=\max _{0 \leq \Omega<\pi}\left|W_{2}\left[e^{j \Omega}\right] S^{a}\left[e^{j \Omega}\right]\right| \leq \varepsilon_{2} .
\end{aligned}
$$

Evidently, these two criteria are similar since the closed-loop transfer functions $T_{r e}^{a}$ and $S^{a}$ are equal. Note, in particular, that boosting the controller gain at a specific frequency simultaneously improves tracking and sensitivity performance at that frequency.

Now consider Figure 1(b), where a sensor delay of $N$ (with $N \geq 1$ ) samples,

$$
F[z]=\frac{1}{z^{N}},
$$

is introduced to the feedback loop. The delayed output signal, denoted $y_{m}[k]$ (where the $m$ subscript denotes "measured"), is fed back to the controller. Obviously the presence of the sensor delay will affect the closedloop performance, possibly causing the loss of "good" performance. A key point is that the closed-loop properties are affected in different ways by the presence of the sensor delay; in particular, the way in which the sensor delay affects tracking is different from the way in which it affects the closed-loop sensitivity. Section 2 considers this issue in more detail, showing that it is possible to design the controller $C^{b}$ to recover tracking performance attained in Figure 1(a), but, because the sensor delay imposes performance limitations, it may not be possible to recover "good" sensitivity performance.

In the radiotherapy project, it was recognized at an early stage that the sensor delay in Figure 1(b) can greatly deteriorate closed-loop performance. The 2DOF control scheme shown in Figure 1(c) was therefore proposed in an attempt to recover the performance of Figure 1(a) (Davison and Hwang, 2003). This scheme, which is a special case of the general observer-predictor controller characterization developed by Mirkin and Raskin (2003), uses an observerbased estimator to estimate the plant output, essentially "cancelling out" the sensor delay. Setting $C^{c}[z]=$ $C^{a}[z]$ recovers the tracking performance from Figure $1(\mathrm{a})$. In the radiotherapy project, this approach (a)

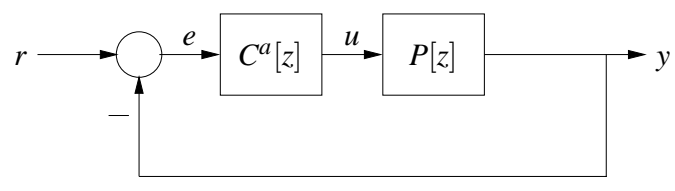

(b)

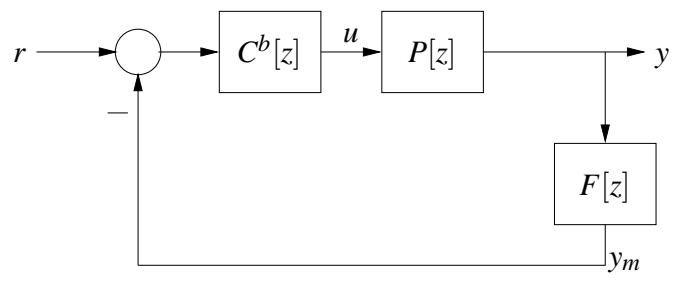

(c)

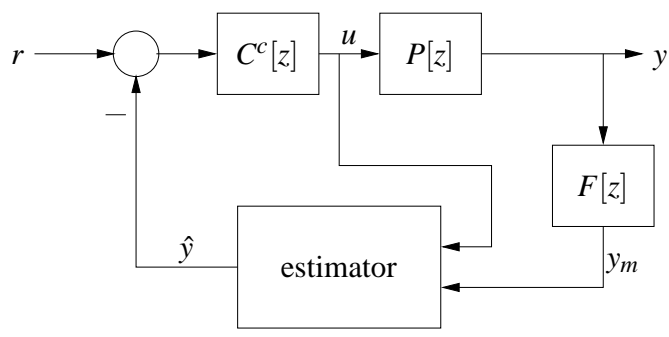

Fig. 1. (a) Basic 1-DOF control loop with no sensor delay; (b) inclusion of a sensor delay in the 1DOF control loop; (c) a 2-DOF control loop, using an observer-based estimator to compensate for the sensor delay.

was taken and used successfully on a prototype experiment. However, little attention was paid to how the estimator was designed since any unbiased stable estimator will effectively "cancel" the sensor delay. Later, an attempt to deal with plant model uncertainty motivated the question that is addressed in Section 3: is it possible to design the estimator to recover the sensitivity properties of Figure 1(a)? Intuitively, some aspect of closed-loop performance must be impaired by the delay, and one would expect the degree of impairment to increase as the length of the sensor delay increases. The main contribution of Section 3 is to show that this intuition is correct; specifically, the sensitivity properties of the 2-DOF setup in Figure 1(c) are examined, and limitations on the achievable sensitivity are derived as a function of the length of the sensor delay. Interestingly, the performance limitation is very similar to that derived in the 1-DOF setup in Figure 1(b).

The 2-DOF delay compensation approach in Figure $1(\mathrm{c})$ is reminiscent of the Smith predictor and its modern descendents (e.g., see (Smith, 1957) and, for a more complete and modern report, (Furukawa and Shimenura, 1983)). The main difference between the setup in this paper and the setup in the Smith predictor literature is that the time delay appears in the feedback loop in the former and in the feedforward path in the latter. Although outside the scope of this paper, the methods used here can be applied to 
derive similar performance limitations for the Smith predictor framework. It should also be pointed out that this work is connected with other research into performance limitations where time delays, typically in a continuous-time 1-DOF framework, are explicitly dealt with (e.g., see (Looze and Freudenberg, 1991) or (Middleton, 1991)). To the best of the authors' knowledge, none of the existing performance limitation results apply to the 2-DOF setup in Figure 1(c), so the contributions of this paper are novel in that respect; however, closely related work includes that of Chen et al. (2000) and Toker et al. (2002).

\section{ANALYSIS OF THE 1-DOF SETUP}

Turn back to the 1-DOF feedback loop in Figure 1(b). The closed-loop transfer functions reflecting the performance criteria of interest are modified from those related to Figure 1(a) as follows (where the superscript $b$ connects the transfer functions with Figure 1(b), and where frequency arguments are omitted for clarity):

- Tracking performance is now measured by the magnitude of

$$
T_{r e}^{b}=\frac{1}{1+P C^{b} F}+\frac{P C^{b}(F-1)}{1+P C^{b} F},
$$

where $e:=r-y$ is no longer the output of the first summer.

- The sensitivity of $T_{r y}^{b}$ with respect to perturbations in the plant, $P$, is now measured by the magnitude of

$$
S^{b}:=\frac{\partial T_{r y}^{b}}{\partial P} \frac{P}{T_{r y}^{b}}=\frac{1}{1+P C^{b} F} .
$$

Note that $T_{r e}^{b}$ differs from $S^{b}$.

The main objective of this section is to determine to what degree it is possible, by designing controller $C^{b}$, to recover the closed-loop performance obtained in Figure 1(a). The following theorem establishes that it is possible to design $C^{b}$ to recover tracking performance:

Theorem 1: The controller

$$
C^{b}=\frac{C^{a}}{1+P C^{a}(1-F)}
$$

stabilizes the closed-loop system in Figure 1(b) and results in "good" tracking, i.e.,

$$
\left\|W_{1} T_{r e}^{b}\right\|_{\infty} \leq \varepsilon_{1} .
$$

Proof: Since $P[z]$ is stable, the set of all stabilizing controllers is

$$
\left\{\frac{Q^{b}}{1-P F Q^{b}}: Q^{b} \text { is stable and proper }\right\},
$$

where $Q^{b}$ is the so-called Youla parameter (Doyle $e t$ $a l ., 1992)$. The controller (5) is stabilizing if it equals one of the controllers in (7). In fact, it is easy to show that controller (5) corresponds to the Youla parameter

$$
Q^{b}=\frac{C^{a}}{1+P C^{a}}
$$

which is stable and proper since $C^{a}$ is assumed to be a stabilizing controller for Figure 1(a). Therefore (5) is stabilizing.

To show that "good" tracking is achieved, compute $T_{r e}^{b}$ in (3) for the controller (5):

$T_{r e}^{b}=\frac{1}{1+P C^{b} F}+\frac{P C^{b}(F-1)}{1+P C^{b} F}=\cdots=\frac{1}{1+P C^{a}}=T_{r e}^{a}$.

In other words, the tracking performance of Figure 1(a) is recovered perfectly, and therefore (1) implies that (6) is satisfied.

Although it is possible to recover tracking performance, sensitivity with respect to plant model uncertainty is fundamentally constrained by the sensor delay, as the following theorem indicates:

Theorem 2: Let $\mathcal{S}$ denote the set of stable, proper transfer functions. Define

$$
\gamma_{N}:=\min _{Q \in \mathcal{S}}\left\|W_{2}-F Q\right\|_{\infty}
$$

Then $\gamma_{N}$ is a monotonically increasing function of $N$. Moreover, for any stabilizing controller in Figure 1(b), sensitivity is limited by $\gamma_{N}$ :

$$
\left\|W_{2} S^{b}\right\|_{\infty} \geq \gamma_{N}
$$

Proof: Standard model-matching theory results will be exploited. In particular, the fact that the solution to $\min _{Q \in \mathcal{S}}\left\|T_{1}-T_{2} Q\right\|_{\infty}$ (for given $T_{1}, T_{2} \in \mathcal{S}$ ) can be found by forming an equivalent interpolation problem on $Q$ (where the interpolation constraints depends only on $T_{2}$ ) is used. A good summary of the continuous-time SISO model-matching problem is provided in (Doyle et al., 1992); the discrete-time problem can be converted to an equivalent continuoustime problem using a bilinear transformation.

Start off by using (4) and (7) to determine that, for any particular stabilizing controller $C^{b}$ with Youla parameter $Q^{b}$ and closed-loop sensitivity $S^{b}$, the following holds:

$$
W_{2} S^{b}=W_{2} \frac{1}{1+P C^{b} F}=W_{2}-W_{2} P F Q^{b} .
$$

Hence,

$$
\begin{aligned}
\left\|W_{2} S^{b}\right\|_{\infty} & \geq \min _{Q \in \mathcal{S}}\left\|W_{2}-W_{2} P F Q\right\|_{\infty} \\
& \geq \min _{Q \in \mathcal{S}}\left\|W_{2}-F Q\right\|_{\infty} \\
& =\gamma_{N} .
\end{aligned}
$$

The second inequality above follows because the removal of $W_{2} P$ from (10) reduces the number of interpolation constraints on $Q$, thus decreasing the best achievable minimum. To argue that $\gamma_{N}$ is an increasing 


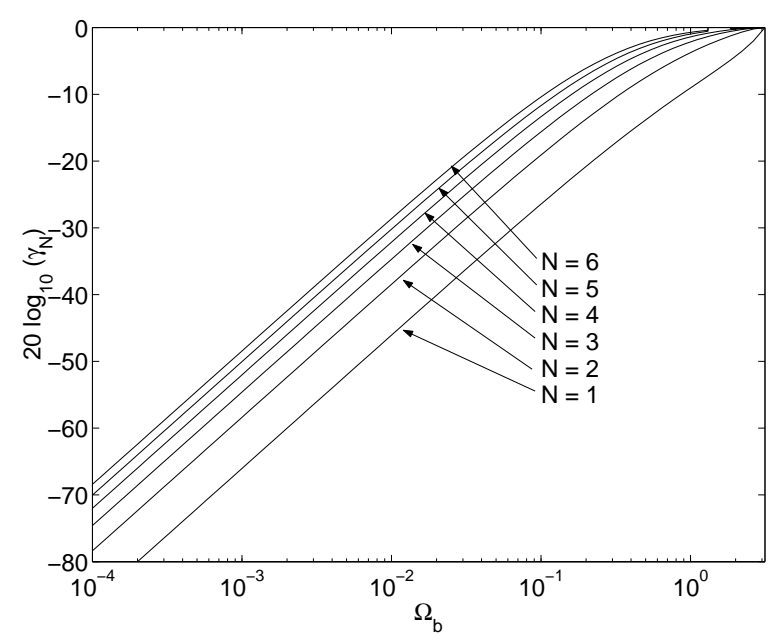

Fig. 2. Discrete-time sensitivity performance limitation curves for weighting function (11).

function of $N$, again an appeal is made to modelmatching theory: since $F[z]=1 / z^{N}$, the only effect of increasing $N$ to $N+1$ is to include an extra pole at $z=0$, which results in an extra interpolation constraint on $Q[z]$, which, in turn, increases the best achievable minimum.

Note that $\gamma_{N}$ depends on the weighting function $W_{2}$, but not on the plant, $P$. For a specific choices of weight, it is possible to numerically evaluate $\gamma_{N}$, thereby quantifying the sensitivity performance limitation. For example, a useful choice of weighting function is the first-order low-pass filter

$$
W_{2}[z]=\frac{z \tan \left(\Omega_{b} / 2\right)+\tan \left(\Omega_{b} / 2\right)}{z\left(\tan \left(\Omega_{b} / 2\right)+1\right)+\left(\tan \left(\Omega_{b} / 2\right)-1\right)},
$$

where $\Omega_{b}\left(0<\Omega_{b}<\pi\right)$ is the bandwidth, as measured by the $-3 \mathrm{~dB}$ frequency. Using standard computational algorithms, the solution to the model-matching problem (8) was computed for different values of $N$ as $\Omega_{b}$ varies from 0 to $\pi$. Figure 2 shows the resulting values of $\gamma_{N}$. The curves show explicitly that there are bounds on the achievable sensitivity in the face of sensor delay, and also that the bounds get progressively worse as the system bandwidth, $\left(\Omega_{b}\right)$ or the sensor delay $(N)$ increases. These curves can be used quantitatively: for example, if $N=1$ (i.e., a sensor delay of one sample), and $\varepsilon_{2}=0.01$ (i.e., sensitivity must be no more than $1 \%$ at all frequencies within the system bandwidth), then the (discrete-time) system bandwidth must satisfy $\Omega_{b}<0.02$; if $N=2$, the bandwidth bound worsens to $\Omega_{b}<0.009$, etc.

In summary, although it is possible to recover tracking performance in the presence of sensor delay, there is a fundamental limitation on the sensitivity performance level that can be obtained, and the limitation worsens as the sensor delay increases. It is next considered what, if anything, can be gained by using an estimator to "cancel out" the sensor delay.

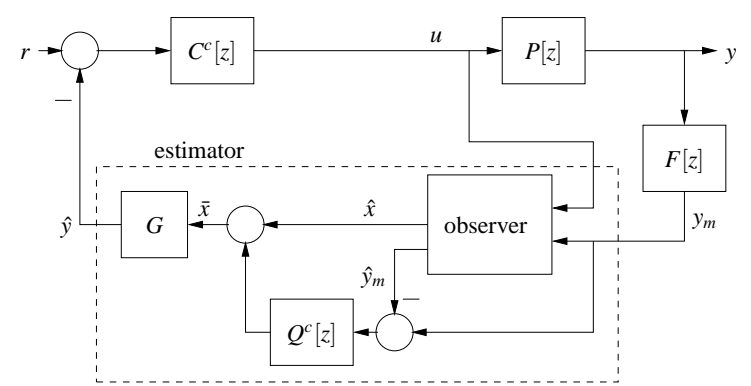

Fig. 3. The 2-DOF control scheme from Figure 1(c), with details of the estimator included.

\section{ANALYSIS OF THE 2-DOF SETUP}

Following previous notation, superscript $c$ is used to connect closed-loop transfer functions with Figure 1(c), and the sensitivity function of interest is now

$$
S^{c}:=\frac{\partial T_{r y}^{c}}{\partial P} \frac{P}{T_{r y}^{c}} .
$$

Before proceeding with the analysis, it is necessary to introduce minimal state-space realizations for the plant, delay, and controller shown in Figure 1(c). Let $P[z]$ have state $x_{p} \in \Re^{n_{p}}$ with state-space matrices $\left(A_{p}, B_{p}, C_{p}, 0\right)$, let $F[z]$ have state $x_{f} \in \mathfrak{R}^{n_{f}}$ with matrices $\left(A_{f}, B_{f}, C_{f}, D_{f}\right)$, and let $C^{c}[z]$ have state $x_{c} \in \mathfrak{R}^{n_{c}}$ with matrices $\left(A_{c}, B_{c}, C_{c}, D_{c}\right)$. Finally, let $m=n_{p}+n_{f}$.

The estimator in Figure 1(c) is assumed to be LTI, stable, and unbiased. Using the results from Goodwin and Middleton (1989), the class of all such estimators can be parameterized by a stable proper (vector) transfer function. Figure 3 shows details of the estimator parameterization, where the estimator parameter is denoted $Q^{c}$. The "observer" shown in the dashed box in Figure 3 is any Luenberger observer, itself a stable unbiased estimator. The observer provides the preliminary estimate $\hat{x}$, where $x$ is the combined state of $P[z]$ and $F[z]$ :

$$
x:=\left[\begin{array}{c}
x_{p} \\
x_{f}
\end{array}\right] \in \Re^{m}
$$

The estimator uses $\hat{x}$ and a filtered version of the innovation process, $y_{m}-\hat{y}_{m}$, to generate another estimate of $x$, this time denoted ${ }^{-} x$. Goodwin and Middleton (1989) prove that any unbiased stable estimator can be generated by an appropriate choice of $Q^{c}$ (for example, the estimate $\hat{x}$ can be recovered using $Q^{c}=0$ ), and any choice of $Q^{c}$ generates an unbiased stable estimator. The final output of the estimator in Figure 3 is an estimate of $y$, computed by $\hat{y}=G^{-} x$ where

$$
G:=\left[\begin{array}{ll}
C_{p} & 0
\end{array}\right] .
$$

Note that $G$ is nonzero since $P[z]$ is not the zero transfer function.

Due to the structure of the estimator, the estimate $\hat{y}$ approaches $y$ as $k \rightarrow \infty$. This implies that the separation principle holds, which, in turn, implies that the 
controller $C^{c}=C^{a}$ recovers the tracking performance associated with Figure 1(a). The following theorem establishes this more formally:

Theorem 3: Consider the control system in Figure 3 with $C^{c}=C^{a}$, with the observer designed to be stable, and with $Q^{c}$ equal to any stable proper (vector) transfer function. Then the closed-loop system is stable and "good" tracking performance is achieved, that is,

$$
\left\|W_{1} T_{r e}^{c}\right\|_{\infty} \leq \varepsilon_{1},
$$

where $e:=r-y$ is the tracking error.

Proof The closed-loop equations for Figure 3 are required. To this end, first find a state-space realization of the cascaded system $F P$ :

$$
\begin{aligned}
x[k+1] & =\hat{A} x[k]+\hat{B} u[k] \\
y_{m}[k] & =\hat{C} x[k],
\end{aligned}
$$

where

$$
\hat{A}=\left[\begin{array}{cc}
A_{p} & 0 \\
B_{f} C_{p} & A_{f}
\end{array}\right], \hat{B}=\left[\begin{array}{c}
B_{p} \\
0
\end{array}\right], \hat{C}=\left[\begin{array}{ll}
D_{f} C_{p} C_{f}
\end{array}\right] .
$$

Note that $\hat{A}$ is Schur stable (i.e., all eigenvalues are inside the unit circle) since $F$ and $P$ are assumed to be stable.

Next, focus on the dashed box in Figure 3, that is, on the estimator portion of the control scheme. The observer equations, with gain $H$, are

$$
\begin{aligned}
\hat{x}[k+1] & =(\hat{A}-H \hat{C}) \hat{x}[k]+\hat{B} u[k]+H y_{m}[k] \\
\hat{y}_{m}[k] & =\hat{C} \hat{x} .
\end{aligned}
$$

It is assumed that the observer is designed to be stable, that is, $H$ is chosen such that $(\hat{A}-H \hat{C})$ is Schur stable. (Since $\hat{A}$ is Schur stable, the choice $H=0$ can be used, for example.) At this point, introduce $x_{q}$ as the state of $Q^{c}$, and denote a state-space realization of $Q^{c}$ by $\left(A_{q}, B_{q}, C_{q}, D_{q}\right)$. Also define $e_{x}:=x-\hat{x}$, and form the stacked state

$$
x_{e s t}:=\left[\begin{array}{l}
e_{x} \\
x_{q}
\end{array}\right] .
$$

A straightforward calculation reveals that the estimator satisfies

$$
\begin{aligned}
& x_{e s t}[k+1]=\tilde{A} x_{e s t}[k] \\
& y[k]-\hat{y}[k]=\tilde{C} x_{e s t}[k],
\end{aligned}
$$

where

$$
\tilde{A}=\left[\begin{array}{cc}
\hat{A}-H \hat{C} & 0 \\
-B_{q} \hat{C} & A_{q}
\end{array}\right], \tilde{C}=\left[G\left(I-D_{q} \hat{C}\right) G C_{q}\right] .
$$

Note that $\tilde{A}$ is Schur stable since each of $(\hat{A}-H \hat{C})$ and $A_{q}$ is Schur stable. Consequently, $x_{e s t}[k] \rightarrow 0$, and therefore $\hat{y}[k] \rightarrow y[k]$, as $k \rightarrow \infty$.

The closed-loop equations for Figure 3 can now be written down. In terms of the closed-loop system state vector

$$
x_{c l s}:=\left[\begin{array}{c}
x_{c} \\
x_{p} \\
x_{e s t} \\
x_{f}
\end{array}\right],
$$

the closed-loop equations are

$$
\begin{aligned}
x_{c l s}[k+1] & =A_{c l s} x_{c l s}[k]+B_{c l s} r[k] \\
y[k] & =C_{c l s} x_{c l s}[k],
\end{aligned}
$$

where

$$
\begin{gathered}
A_{c l s}=\left[\begin{array}{cccc}
A_{c} & -B_{c} C_{p} & B_{c} \tilde{C} & 0 \\
B_{p} C_{c} & A_{p}-B_{p} D_{c} C_{p} & B_{p} D_{c} \tilde{C} & 0 \\
0 & 0 & \tilde{A} & 0 \\
0 & B_{f} C_{p} & 0 & A_{f}
\end{array}\right], \\
B_{c l s}=\left[\begin{array}{c}
B_{c} \\
B_{p} D_{c} \\
0 \\
0
\end{array}\right], C_{c l s}=\left[\begin{array}{llll}
0 & C_{p} & 0 & 0
\end{array}\right] .
\end{gathered}
$$

Evidently, the closed-loop eigenvalues are the union of those of $A_{f}$ (which is stable since $F[z]$ is stable), those of $\tilde{A}$ (which is stable, as mentioned above), and those of

$$
\bar{A}:=\left[\begin{array}{cc}
A_{c} & -B_{c} C_{p} \\
B_{p} C_{c} & A_{p}-B_{p} D_{c} C_{p}
\end{array}\right]
$$

(which is stable since $\bar{A}$ is the closed-loop " $A$ " matrix of the stable feedback system in Figure 1(a)). Hence, the feedback system in Figure 3 is stable.

Lastly, one can use the above closed-loop matrices to show that $T_{r y}^{c}=C_{c l s}\left(z I-A_{c l s}\right)^{-1} B_{c l s}=\cdots=P C^{c} /(1+$ $\left.P C^{c}\right)$. Hence, $T_{r e}^{c}=1-T_{r y}^{c}=1 /\left(1+P C^{a}\right)=T_{r e}^{a}$. That is, the tracking performance of Figure 1(a) is recovered exactly.

Having established that, in Figure 3, "good" tracking performance is achieved with $C^{c}=C^{a}$ and with $Q^{c}$ equal to any stable proper (vector) transfer function, the closed-loop sensitivity aspect of performance is now addressed:

Theorem 4: Consider the control system in Figure 3 with $C^{c}=C^{a}$, with the observer designed to be stable, and with $Q^{c}$ equal to any stable proper (vector) transfer function. Then closed-loop sensitivity is limited by $\gamma_{N}$, as defined in (8). That is,

$$
\left\|W_{2} S^{c}\right\|_{\infty} \geq \gamma_{N}
$$

Proof: Since $P[z]$ is assumed to be stable, the observer gain $H=0$ can (and will) be used; this choice can be made without loss of generality, and it simplifies the calculations considerably. In the special case $H=0$, the block diagram in Figure 3 can be redrawn, after some simple block manipulation, as in Figure 4. In Figure 4 , a distinction is made between the actual plant, denoted $P[z]$, and the model of the plant used in the observer, denoted $P_{0}[z]$. This distinction is required to compute $S^{c}$, where it is imagined that $P[z]$ is 


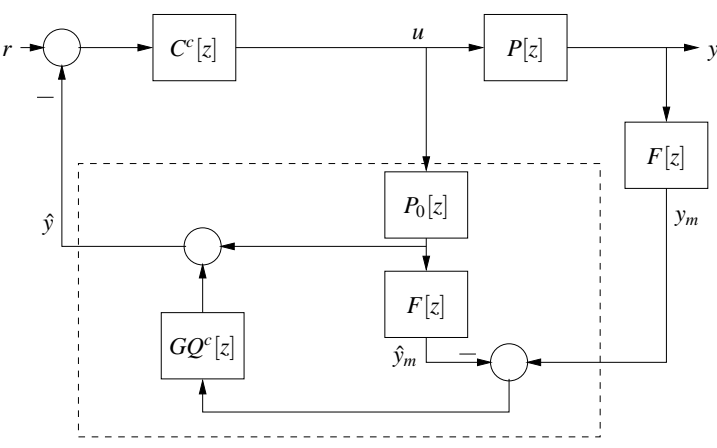

Fig. 4. An equivalent representation of Figure 3 in the special case $H=0$.

perturbed around the nominal value $P_{0}[z]$. A straightforward calculation shows that, for Figure 4,

$$
\frac{\partial T_{r y}^{c}}{\partial P} \frac{P}{T_{r y}^{c}}=\frac{1+C^{c} P_{0}\left(1-G Q^{c} F\right)}{1+C^{c} P_{0}+C^{c} F G Q^{c}\left(P-P_{0}\right)},
$$

and therefore the sensitivity of $T_{r y}^{c}$ with respect to perturbations in $P$, evaluated at $P=P_{0}$, is

$$
S^{c}=\frac{1+C^{c} P_{0}\left(1-G Q^{c} F\right)}{1+C^{c} P_{0}}=1-T_{r y}^{c} G Q^{c} F .
$$

(Note that $T_{r y}^{c}=P_{0} C^{c} /\left(1+P_{0} C^{c}\right)$ is the closed-loop transfer function from $r$ to $y$ when $P=P_{0}$.) Denote the set of stable and proper $m \times 1$ vector transfer functions by $\mathcal{S}^{m}$. Then, for any particular choice of $Q^{c} \in \mathcal{S}^{m}$ with corresponding value of $S^{c}$ in (14), proceed as in the proof of Theorem 2:

$$
\begin{aligned}
\left\|W_{2} S^{c}\right\|_{\infty} & \geq \min _{Q^{m} \in \mathcal{S}^{m}}\left\|W_{2}-W_{2} T_{r y}^{c} G Q^{m} F\right\|_{\infty} \\
& =\min _{Q \in \mathcal{S}}\left\|W_{2}-W_{2} T_{r y}^{c} Q F\right\|_{\infty} \\
& \geq \min _{Q \in \mathcal{S}}\left\|W_{2}-F Q\right\|_{\infty} \\
& =\gamma_{N} .
\end{aligned}
$$

(The first equality above follows from the fact that $G$ in (12) is nonzero.) This completes the proof.

Theorem 4 establishes that, although "good" tracking is achievable, closed-loop sensitivity in Figure 3 is fundamentally constrained by the presence of the sensor delay. Interestingly, the performance bound is the same as in the 1-DOF setup (see Theorem 2). Of course, Theorem 2 and Theorem 4 are different since Theorem 2 applies to the 1-DOF configuration where the free parameter is the Youla parameter, $Q^{b}$, and Theorem 4 applies to the 2-DOF configuration where the free parameter is the estimator parameter, $Q^{c}$. In Theorem 2, the performance limitation on sensitivity applies without regard for tracking performance, but in Theorem 4 the performance limitation holds under the constraint that "good" tracking performance is achieved.

As discussed in the 1-DOF setting, a reasonable and typical choice for $W_{2}[z]$ is a first-order filter of the form (11). Since the bounds in Theorems 2 and 4 are identical, the quantitative bounds provided in Figure 2 apply for both the 2-DOF setup and the 1-DOF setup.

\section{CONCLUSIONS}

In this work it has been proven that, consistent with intuition, there are fundamental performance limitations associated with feedback sensor delays. Theorems 2 and 4 establish bounds on the achievable closed-loop sensitivity in the case where the plant is open-loop stable, in the 1-DOF and 2-DOF control configurations, respectively. The techniques of this work can also be used to prove that there are limitations on disturbance rejection in the face of sensor delays, although these results are omitted due to space limitations. It may be of interest to note that the methods in this paper can be adopted to the continuous-time case, although the estimator formulation is naturally more complicated. Finally, in the case where the plant is open-loop unstable, it is to be expected that the achievable performance (including tracking) should worsen; finding meaningful performance bounds in this situation is a topic of current investigation.

\section{REFERENCES}

Chen, Jie, Li Qiu and Onur Toker (2000). Limitations on maximal tracking accuracy. IEEE Transactions on Automatic Control 45(2), 326-331.

Davison, D. E. and E. S. Hwang (2003). Automating radiotherapy cancer treatment: Use of multirate observer-based control. In: Proceedings of the American Control Conference. Denver, CO.

Doyle, John C., Bruce A. Francis and Allen R. Tannenbaum (1992). Feedback Control Theory. Macmillan. New York, NY.

Furukawa, Toshio and Etsujiro Shimenura (1983). Predictive control for systems with time delay. International Journal of Control 37(2), 399-412.

Goodwin, G.C. and R.H. Middleton (1989). The class of all stable unbiased state estimators. Systems \& Control Letters 13, 161-163.

Looze, Douglas P. and James S. Freudenberg (1991). Limitations of feedback properties imposed by open-loop right half plane poles. IEEE Transactions on Automatic Control 36(6), 736-739.

Middleton, R. H. (1991). Trade-offs in linear control system design. Automatica 27(2), 281-292.

Mirkin, L. and N. Raskin (2003). Every stabilizing dead-time controller has an observer-predictorbased structure. Automatica 39, 1747-1754.

Smith, Otto J.M. (1957). Closer control of loops with dead time. Chemical Engineering Progress 53(5), 217-219.

Toker, Onur, Jie Chen and Li Qiu (2002). Tracking performance limitations in LTI multivariable discrete-time systems. IEEE Transactions on Circuits and Systems-I 49(5), 657-670. 